Artículo científico

(Original paper)

\title{
MICROCENTRUM TECACTLI, NUEVA ESPECIE DE PHANEROPTERIDAE (ORTHOPTERA: TETTIGONIOIDEA) DEL NORESTE DE MÉXICO
}

\author{
MICROCENTRUM TECACTLI, A NEW SPECIES OF PHANEROPTERIDAE \\ (ORTHOPTERA: TETTIGONIOIDEA) FROM NORTHEASTERN MEXICO
}

\author{
GEOVANY J. FERNÁNDEZ-AZUARA, AURORA Y. ROCHA-SÁNCHEZ, LUDIVINA BARRIENTOS- \\ LOZANO*, Alfonso CORREA-SANDOVAL, PEdro AlMAGUER-SIERRA \\ Tecnológico Nacional de México-Instituto Tecnológico de Ciudad Victoria-División de estudios de Posgrado e Investigación. \\ Boulevard Emilio Portes Gil No.1301. Ciudad Victoria, Tamaulipas, México. C.P. 87010 \\ <biol.geofa@hotmail.com>, <auro_3110@hotmail.com>, <ludivinab@yahoo.com>, <alf_correas@ hotmail.com>, \\ <almagavetec@hotmail.com> \\ *Autor de correspondencia: <ludivinab@yahoo.com>
}

Recibido: 11/04/2018; aceptado: 06/09/2018; publicado en línea: 13/11/2018

Editor responsable: Magdalena Cruz

Fernández-Azuara, G. J., Rocha-Sánchez, A. Y., Barrientos-Lozano, L., Correa-Sandoval, A., Almaguer-Sierra, P. (2018) Microcentrum tecactli, nueva especie de Phaneropteridae (Orthoptera: Tettigonioidea) del noreste de México. Acta Zoológica Mexicana (nueva serie), 34, 1-14. https://doi.org/10.21829/azm.2018.3412133

RESUMEN. Se describe Microcentrum tecactli n. sp., Barrientos-Lozano, Fernández-Azuara \& RochaSánchez, 2018, de ejemplares recolectados en la huasteca hidalguense en México. Microcentrum tecactli n. sp., presenta afinidades con Microcentrum rhombifolium (Saussure, 1859). No obstante, difiere de ésta en caracteres morfológicos y acústicos. Los machos producen dos tipos de señales acústicas: i) el canto de llamado que consiste de un siseo regular, de una sola sílaba, cuya duración promedio es de $155 \pm 38 \mathrm{~ms}$ (78-212 ms) (3 machos; $\mathrm{n}=29$ muestras de un min cada una), con intervalo entre sílabas (Intervalo Mayor=IMa) de $1483 \pm 364 \mathrm{~ms}(115-2394 \mathrm{~ms})$. El rango de frecuencia es de 4 a $>30 \mathrm{kHz}$ y la frecuencia pico (fi) de 6-16 kHz. La tasa de repetición de sílabas es de 48 44.36/min (43-55; $\mathrm{n}=10$ muestras de un min cada una). ii) Secuencias de pulsaciones/tics de dos frases-pares con duración promedio de $7.53 \pm 0.16 \mathrm{~s} \mathrm{(3}$ machos; $n=5$ secuencias-pares de pulsaciones/tics); la duración promedio de las frases 1 y 2 es igual en cada secuencia, $2.3 \pm 0 \mathrm{~s}$. El número de pulsaciones por frase es también igual en ambas frases, en cada secuencia: 29 \pm 0 . El intervalo mayor entre secuencias de pulsaciones de dos frases-pares es de $5.37 \pm 2.75$ $\mathrm{s}$. El intervalo menor entre las frases 1 y 2 es de $1.42 \pm 0.12 \mathrm{~s}(1.27-1.51 \mathrm{~s})$. El rango de frecuencia y la frecuencia pico $(f i)$ coinciden con las del canto de llamado. La tasa de repetición de secuencias de pulsaciones-pares es de $0.1 / \mathrm{s}(6 / \mathrm{min})$, en cuatro secuencias de pulsaciones-pares la tasa de repetición de pulsaciones/s en las frases 1 y 2 fue igual, 12.6 en cada una.

Palabras clave: Biodiversidad; Ensifera; Bioacústica; Phaneropterinae

Fernández-Azuara, G. J., Rocha-Sánchez, A. Y., Barrientos-Lozano, L., Correa-Sandoval, A., Almaguer-Sierra, P. (2018) Microcentrum tecactli, a new species of Phaneropteridae (Orthoptera: Tettigonioidea) from Northeastern Mexico. Acta Zoológica Mexicana (nueva serie), 34, 1-14. https://doi.org/10.21829/azm.2018.3412133 
ABSTRACT. Microcentrum tecactli n. sp., Barrientos-Lozano, Fernández-Azuara \& Rocha-Sánchez, 2018, is described from specimens collected in the Huasteca region in the State of Hidalgo Mexico. Microcentrum tecactli exhibits affinities with Microcentrum rhombifolium (Saussure, 1859). However, it differs in morphological and acoustic characters. In males the length of the stridulatory file is $4.2 \mathrm{~mm}$ with ca. 62 teeth vs. $4 \mathrm{~mm} \mathrm{ca.} 55$ teeth in M. rhombifolium; the subgenital plate is distally more produced, the styles are shorter and diverging; the cerci are distally narrower, and the internal genitalia is different. Lone males produce two types of acoustic signals: i) a calling song that consists of a regular buzz made up of a single syllable (one Pulse Train Group), average duration 155 $\pm 38 \mathrm{~ms}$ (78-212 ms) (3 males; $\mathrm{n}=29$ samples one min each), with inter-syllable interval (Major Interval=MaI) of $1483 \pm 364 \mathrm{~ms}$ (115-2394 ms). Frequency spectrum is between 4 to $>30 \mathrm{kHz}$, peak frequency $(\mathrm{fi})$ is at $6-16 \mathrm{kHz}$. Syllables repetition rate is $48 \pm 4.36 / \mathrm{min}$ (43-55; $\mathrm{n}=10$ samples one min each). ii) Sequence of ticks made up of two paired-phrases with an average duration of $7.53 \pm 0.16 \mathrm{~s}$ ( 3 males; $n=5$ paired-sequences of ticks); phrases 1 and 2 average duration is similar in each sequence, $2.3 \pm 0 \mathrm{~s}$. The number of pulses per phrase is also the same in both phrases: $29 \pm 0$ each. Major interval duration between sequences of ticks of two paired-phrases is $5.37 \pm 2.75 \mathrm{~s}$. Minor interval duration between phrases 1 and 2 is $1.42 \pm 0.12 \mathrm{~s}(1.27-1.51)$. Frequency range is between 4 to $>30 \mathrm{kHz}$, after $30 \mathrm{kHz}$ the amount of energy is minimal. Dominant frequency spectrum ranges from 4 to $22 \mathrm{kHz}$ and peak frequency (fi) is at 6-16 kHz. Repetition rate of tick-paired sequences is $0.1 / \mathrm{s}(6 / \mathrm{min})$; in four paired-sequences of ticks the repetition rate of pulses/ticks in phrases 1 and 2 was the same, $12.6 / \mathrm{s}$ each one.

Key words: Biodiversity; Ensifera; Bioacoustics; Phaneropterinae

\section{INTRODUCCIÓN}

La subfamilia Phaneropterinae representa actualmente una de las cuatro subfamilias de Phaneropteridae (Ensifera: Tettigonioidea) (Mugleston et al., 2013; Heller et al., 2014; Braun, 2015). Ésta constituye uno de los grupos más diversos en México y en el mundo (Barrientos-Lozano et al., 2013; Heller et al., 2015). De acuerdo con Fontana et al. (2008) y Cigliano et al. (2018), en México ocurren al menos ocho especies del género Microcentrum Scudder, 1862. Entre los caracteres distintivos del género tenemos los siguientes: la cabeza es oval, ancha y robusta; el tubérculo del vertex es prominente, escasamente más ancho que la primera unión antenal, ligeramente surcado; los ojos son ampliamente ovales y prominentes; el protórax es plano o muy ligeramente cóncavo en vista dorsal, el margen anterior es ligeramente cóncavo y el posterior convexo, los lados son casi paralelos, ligeramente más largos que anchos, las carinas laterales son conspicuas, los lóbulos laterales son más largos que anchos, el margen anterior es recto, el ángulo inferior es redondo y curvo, el margen ventral es redondo. Microcentrum presenta mayor afinidad con Phylloptera Serville, 1831. No obstante, pueden diferenciarse de la manera siguiente: en Microcentrum la cabeza es más ancha y robusta que en Phylloptera; las tegmina tienen el área anterior dorsal triangular extendiéndose posteriormente, más allá que en Phylloptera; las tegmina son redondas pero no de forma regular como en Phylloptera, el margen dorsal (interior) es más recto casi hasta la punta, el margen dorsal externo (superior) declina suavemente hacia la punta, de modo que distalmente las tegmina se estrechan y la punta es más angosta que en Phylloptera. Las patas son mucho más cortas que en Phylloptera, especialmente las patas posteriores. En hembras, el ovipositor es muy corto, fuertemente curvo y sin punta. La especie más común en el noreste de México es M. rhombifolium (Saussure, 1859), ésta tiene una amplia distribución geográfica, desde la región oriente y sur de Estados Unidos al centro de México (Cigliano et al., 2018). M. rhombifolium ha sido bien estudiada, incluyendo el comportamiento acústico de los machos y el órgano estridulatorio en las hembras (Fulton, 1933; Spooner, 1968; Nickle \& Carlysle, 1975; Nickle, 1976). De acuerdo con Spooner (1968) y Nickle (1976), en el sistema de comunicación acústica de $M$. rhombifolium los machos solos (no en contacto con machos o hembras conespecíficos) producen dos tipos de sonido: un "siseo" (lisp) y "tics" (ticks), ambos durante el movimiento de cierre de las tegmina. La actividad acústica es mayor durante la noche, aunque también 
cantan durante el día. Nickle (1976) indica que siseos y tics ocurren de forma independiente, no hay relación de unos con otros. Spooner (1968) registró una tasa de siseos $\left(26^{\circ} \mathrm{C}\right)$ de 1 por $3.0 \mathrm{~s}(2.0-4.1)$ $(\mathrm{n}=13)$, la duración de un siseo fue de $25 \pm 3 \mathrm{~ms}(22-30)(\mathrm{n}=10)$. Una serie de tics (una frase) corresponde a golpes de dientes individuales al cerrar el macho las tegmina. Tres frases, de cada uno de dos machos estudiados, promediaron $8,5\left(25^{\circ} \mathrm{C}\right)$ y $8,8\left(25.5^{\circ} \mathrm{C}\right)$ tics por segundo, respectivamente y están compuestas de 22 a 34 tics por frase. Nickle (1976) obtuvo 22-25 tics por secuencia (frase), con una tasa de repetición de $9 \mathrm{tics} / \mathrm{s}\left(25^{\circ} \mathrm{C}\right)$. Diferencias inter-específicas en frecuencia y otros parámetros físicos de la señal acústica en Phaneropterinae han sido estudiadas por varios autores (Nickle \& Carlysle, 1975; Spooner, 1995; Heller et al., 2015, 2017).

En 2015, durante expedición realizada para estudiar los Ensifera en la región huasteca del Estado de Hidalgo, México, se recolectaron varios ejemplares de Phaneropterinae que se asignaron al género Microcentrum. Sin embargo, éstos no corresponden a la descripción de ninguna de las especies que se conocen para el género. Por lo que en el presente trabajo se describe Microcentrum tecactli n. sp., de ejemplares recolectados en San Felipe Orizatlán, Hidalgo, México. M. tecactli n. sp., se compara con $M$. rhombifolium, se proporcionan caracteres de diagnóstico para separar ambas especies y se analiza la señal acústica de los machos comparándola con la señal acústica de M. rhombifolium.

\section{MATERIALES Y MÉTODOS}

Ejemplares adultos de $M$. tecactli $n$. sp., ( $\bigcirc^{\lambda}$ y ㅇ) se recolectaron en el municipio de San Felipe Orizatlán, Hidalgo, México, $21.169319 \mathrm{~N}$ y -98.625783 O, $130 \mathrm{msnm}$, huasteca hidalguense. Éstos se transportaron vivos al laboratorio del Instituto Tecnológico de Cd. Victoria (ITCV) y se mantuvieron separados machos y hembras en jaulas de 30X30X30 cm, para facilitar la producción y grabación de señales acústicas. Como alimento se les proporcionó hojas de durazno y lechuga cada tercer día. Fotografías y medidas de los caracteres de diagnóstico estudiados se tomaron con un microscopio estereoscópico marca MOTIC-SWZ$168739^{\circledR}$ equipado con cámara digital de $10 \mathrm{mp}$ : fastigio del vertex y fastigio frontal (20X), pronoto vista dorsal y lateral (10X), aparato estridulador (10X), peine estridulador (20X), cercos y plato subgenital (10X), cercos-acercamiento (30X), ovipositor (10X). Se estudió el canto de llamado "siseo" y "secuencias de pulsaciones (tics)" de tres machos. Las grabaciones se realizaron por la noche, utilizando una computadora portátil $\mathrm{Hp}$ y un micrófono ultrasónico ULTRAMIC 250K-Dodotronic, frecuencia de muestreo $250 \mathrm{kHz}$, rango de frecuencia hasta $125 \mathrm{kHz}$, temperatura $30 \pm 1^{\circ} \mathrm{C}$, a una distancia de $50 \mathrm{~cm}$. La señal acústica se analizó con el programa Cool Edit Pro 2.1, 16 bits, tamaño FFT 4096. La terminología para describir las señales acústicas puede consultarse en Spooner (1968), Morris \& Walker (1976), Ragge \& Reynolds (1998), Stumpner et al. (2013), Heller et al. (2004, 2015).

Canto de llamado: canto producido por un macho solo, generalmente aislado de otros, cuando está sexualmente maduro.

Sílaba: sonido producido durante un ciclo individual y completo de apertura y cierre de las tegmina.

Pulsación (Pulse): sonido aislado, producido generalmente por la estimulación de uno o varios dientes del peine estridulador.

Secuencia de pulsaciones (=secuencia de tics=frase): en este trabajo, grupos de pulsaciones/tics producidos en serie, las cuales pueden ser resueltas por el oído humano y contadas.

Intervalo Mayor-IMa: intervalo de silencio o casi, que sigue a un tren de pulsaciones de una, dos o más frases y/o sílabas. 
Intervalo Menor-IMe: en este trabajo, intervalo de silencio o casi, que hay entre las frases 1 y 2 en secuencias-pares de pulsaciones/tics. Se midió la duración (segundos=s; o milisegundos=ms) de la señal de llamado "siseo" ( 3 machos; $\mathrm{n}=29$ muestras de un min cada una) y de la secuencia(s) de pulsaciones/tics (3 machos; $n=5$ secuencias-pares de pulsaciones/tics), el Intervalo Mayor (IMa) entre sílabas en la señal de llamado y entre secuencias-pares de pulsaciones, el Intervalo Menor (IMe) entre frases en secuenciaspares de pulsaciones, el espectro de frecuencia y la frecuencia $(f i)$ pico, la tasa de repetición de sílabas por min (t.r sílabas/min) en el canto de llamado y la tasa de repetición de pulsaciones por s y/o por min (t.r pulsaciones/s o min) en secuencias de pulsaciones.

Depósito de material tipo y paratipos. Colección L. Barrientos-Lozano, Tecnológico Nacional de México-Instituto Tecnológico de Cd. Victoria (TecNM-ITCV), Tamaulipas, México.

\section{RESULTADOS}

\section{Microcentrum tecactli n. sp., Barrientos-Lozano, Fernández-Azuara \& Rocha-Sánchez} Figs. 1-16, 37.

Material tipo. Holotipo $\widehat{\partial}$, México, Hidalgo, San Felipe Orizatlán, 130 msnm, 21.169319 N-98.625783 O, 07.X.2015, Fernández-Azuara G. J. Paratipos: $2 \curvearrowright, 2$ ㅇ, mismos datos del material tipo.

Diagnosis. Machos (Figs. 1-10, 37). Similar a M. rhombifolium (Fig. 37 vs. 38), se puede diferenciar de la última especie por los siguientes caracteres: margen anterior del fastigio del vertex, vista frontal, (Fig. 4 vs. 20) fuertemente ondulado (casi recto en M. rhombifolium), ángulos laterales anteriores muy abultados (moderadamente abultados en $M$. rhombifolium); fastigio frontal con margen anterior irregular (semicircular en $M$. rhombifolium), la porción media proyectándose hacia la ondulación media del fastigio del vertex. Longitud del peine estridulador $4.2 \mathrm{~mm}$ con $c a$. 62 dientes $v s$., $4 \mathrm{~mm}$ con $c a$. 55 dientes en $M$. rhombifolium (Fig. 6 vs. 22). Plato subgenital-vista ventral- más largo, $2.4 \mathrm{~mm}$ vs. $1.8 \mathrm{~mm}$ en $M$. rhombifolium (Fig. 10 vs. 26), más evidente en la porción distal; emarginación distal en forma de "u" más amplia que en $M$. rhombifolium, estilos ligeramente más cortos y divergentes (casi rectos en $M$. rhombifolium). Los cercos de $M$. tecactli son basalmente un poco más gruesos y el ápice más angosto (Figs. 7-10 vs. 23-26). Genitalia interna como se muestra en las Figs. 39a, b vs. 40a, b. Hembras (Figs. 11-16), ovipositor más largo $(5.8 v s .5 .2 \mathrm{~mm})$ y más ancho (2.3 vs. $1.9 \mathrm{~mm})$ (Fig. $15 \mathrm{vs}$. 31), plato subgenital distalmente emarginado en "v" vs., redondo en M. rhombifolium (Figs. 16 y 32 ).

Descripción machos (incluye holotipo). Color general del cuerpo verde claro (Figs. 1, 37). Ojos ovales y prominentes (Figs. 2-4). Margen anterior del pronoto (Fig. 2) moderadamente sinuoso, ligeramente cóncavo, carina media principal conspicua, carinas laterales prominentes; lóbulos laterales más largos que anchos, sinus humeral aproximadamente en ángulo recto (Fig. 3). Fastigio del vertex (Fig. 4) con un surco dorsal ancho a lo largo de la línea media, margen anterior ondulado con incisión conspicua en la porción media, ángulos laterales anteriores abultados; fastigio frontal con márgenes irregulares, porción media anterior proyectándose hacia la incisión del fastigio del vertex. Aparato estridulador como se muestra en la Fig. 5. Longitud del peine estridulador $4.2 \mathrm{~mm}$, con ca. 62 dientes (Fig. 6). La longitud de las tegmina se proporciona en la sección de medidas. Cercos como se muestran en las Figs. 7-10. Plato subgenital (Figs. $7-8,10)$ tricarinado, emarginación distal en forma de "u" amplia, estilos de tamaño mediano y divergentes.

Descripción hembras. Similar al macho, solo ligeramente más grandes (Fig. 11). Margen anterior del pronoto sinuoso, moderadamente emarginado; la emarginación es más notoria en la porción media (Figs. 12-13). Fastigio del vertex y frontal como se muestra en la Fig. 14. Ovipositor corto y robusto, valva dorsal con serrulación distal prominente (Fig. 15). Plato subgenital robusto sub-triangular, distalmente "v" emarginado (Figs. 15-16). 

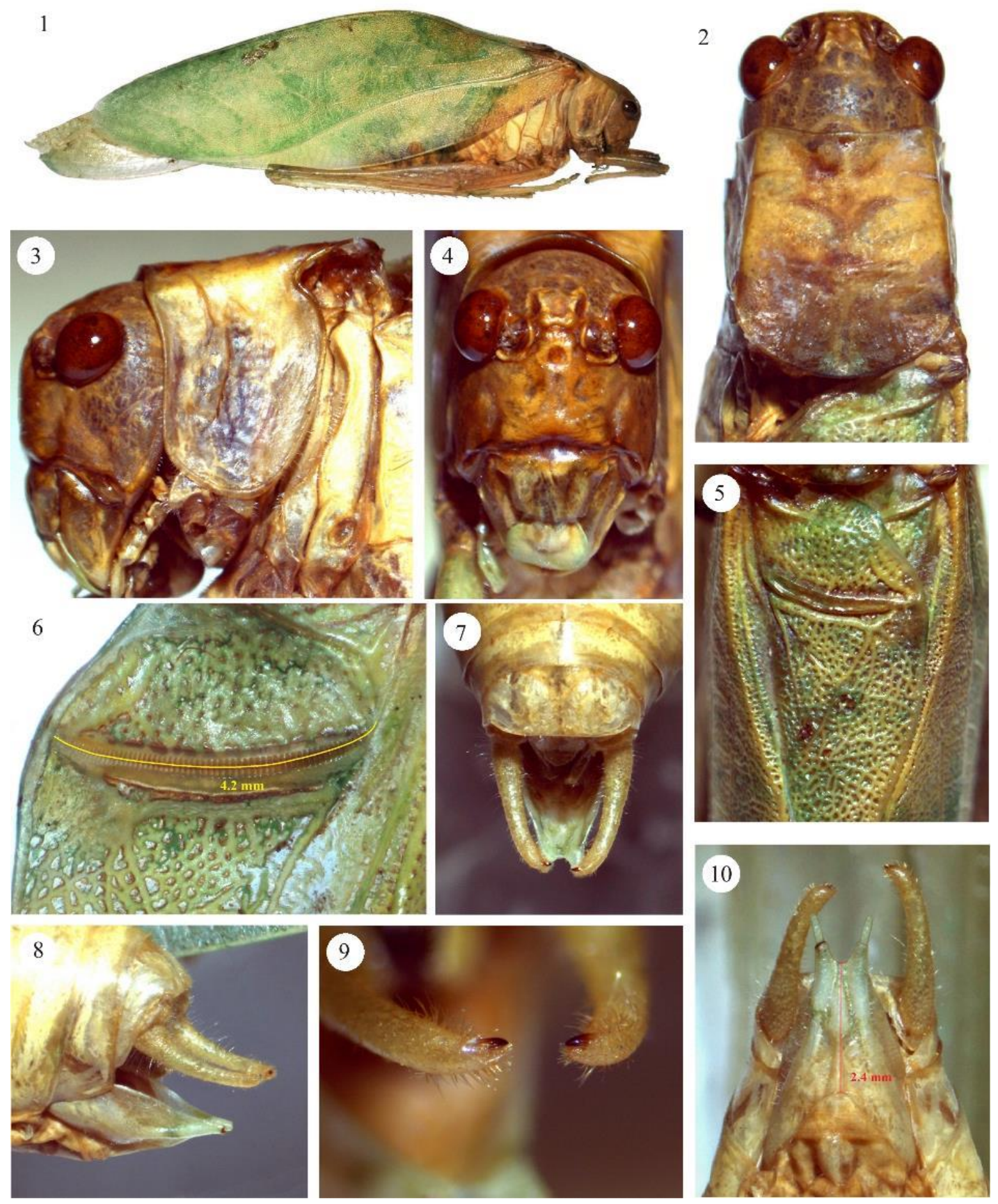

Figuras 1-10. Microcentrum tecactli n. sp. Macho, 1) habitus, 2) cabeza y pronoto, vista dorsal, 3) cabeza y pronoto, vista lateral, 4) fastigio del vertex y fastigio frontal, 5) aparato estridulador, 6) peine estridulador, 7) cercos vista dorsal, 8) cercos y plato subgenital, vista lateral, 9) acercamiento de los cercos, vista dorsal, 10) plato subgenital, vista ventral. 

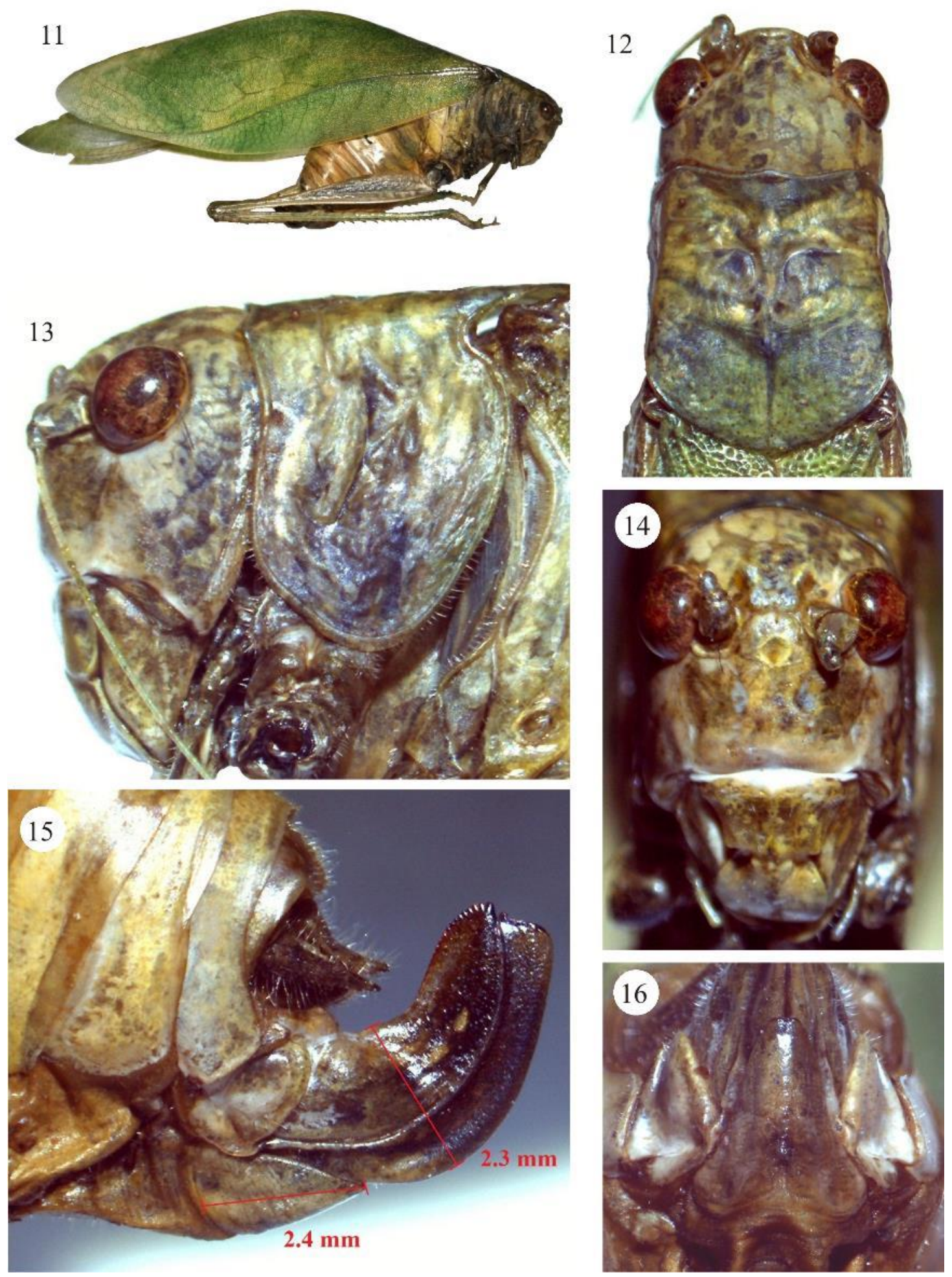

Figuras 11-16. Microcentrum tecactli $\mathrm{n}$. sp. Hembra, 11) habitus, 12) cabeza y pronoto, vista dorsal, 13) cabeza y pronoto, vista lateral, 14) fastigio del vertex y fastigio frontal, 15) ovipositor, plato subgenital y lóbulo de ovipositor, vista lateral, 16) plato subgenital, vista ventral. 

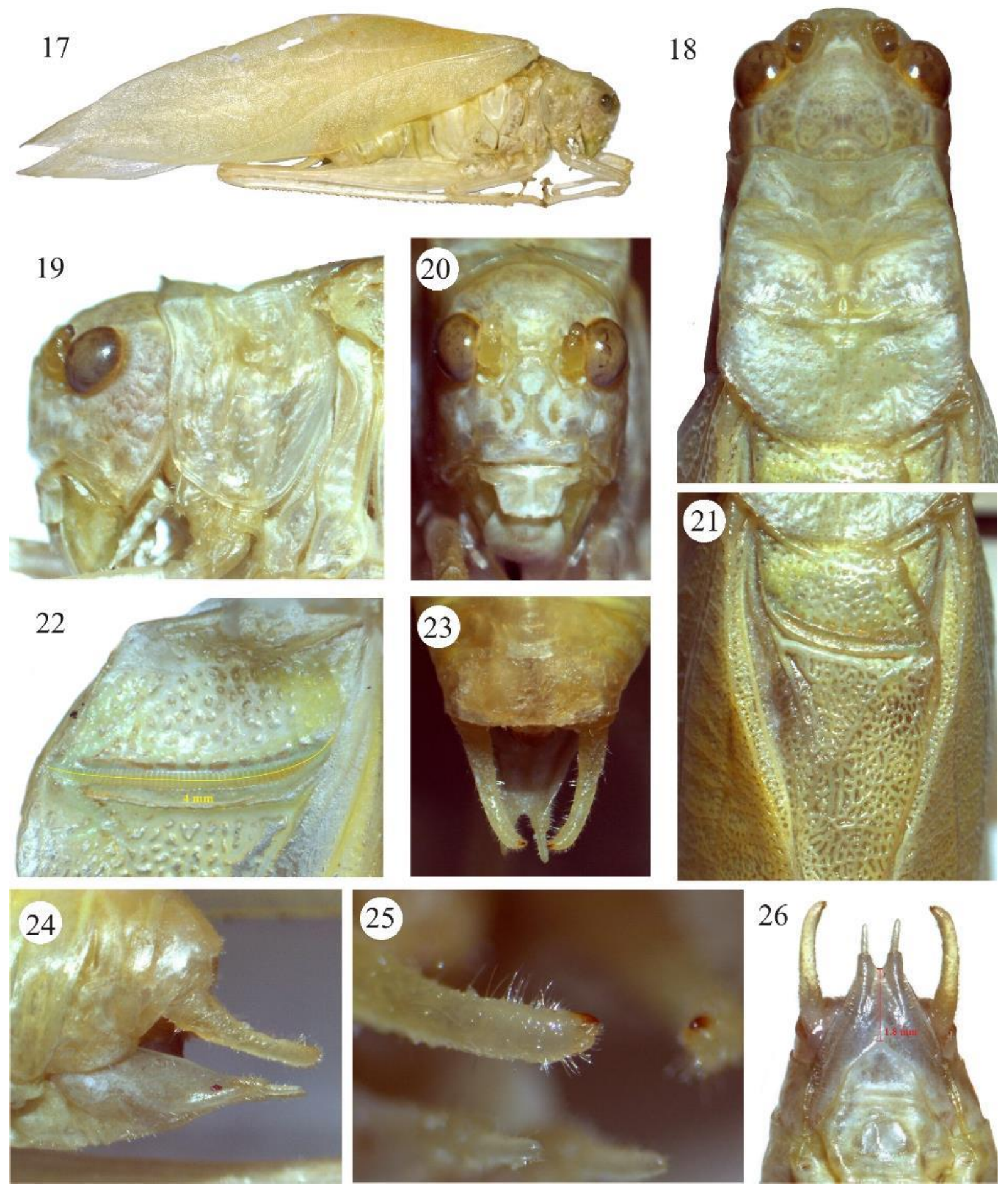

Figuras 17-26. Microcentrum rhombifolium. Macho, 17) habitus, 18) cabeza y pronoto, vista dorsal, 19) cabeza y pronoto, vista lateral, 20) fastigio del vertex y fastigio frontal, 21) aparato estridulador, 22) peine estridulador, 23) cercos vista dorsal, 24) cercos y plato subgenital, vista lateral, 25) acercamiento de los cercos, vista dorsal, 26) plato subgenital, vista ventral. 

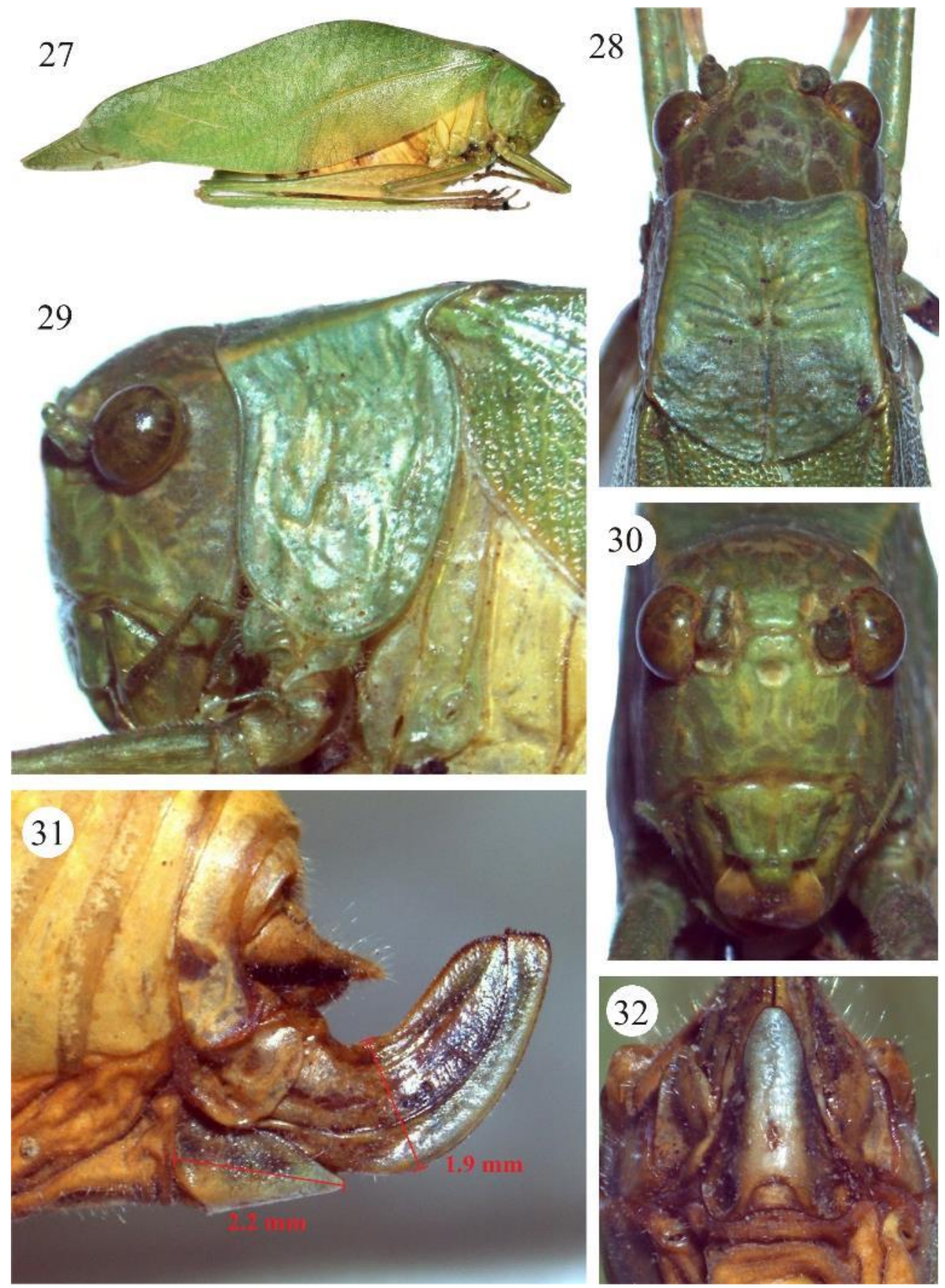

Figuras 27-32. Microcentrum rhombifolium. Hembra, 27) habitus, 28) cabeza y pronoto, vista dorsal, 29) cabeza y pronoto, vista lateral, 30) fastigio del vertex y fastigio frontal, 31) ovipositor, plato subgenital y lóbulo del ovipositor, vista lateral, 32) plato subgenital, vista ventral.

Medidas (mm). Machos. Longitud del cuerpo, desde el margen anterior-porción media del vertex al final de las tegmina, 58. Longitud del pronoto, 5. Anchura máxima pronoto, 5.5. Longitud de las tegmina, 42.7. Anchura máxima de las tegmina, 14. Longitud del femur anterior, 6. Longitud del femur medio, 9.4. Longitud del femur posterior, 23. Hembras. Longitud del cuerpo, desde margen anterior-porción media del vertex al final de las tegmina (tegmina ligeramente dañadas en el ápice), 57. Longitud del pronoto, 5.9. Anchura máxima pronoto, 5.5. Longitud de las tegmina, 43.2. Anchura máxima de las tegmina, 14. 
Longitud del femur anterior, 6. Longitud del femur medio, no disponible (ND). Longitud del femur posterior, 23. Longitud del ovipositor, 5.8. Anchura máxima ovipositor, 2.3. Longitud del plato subgenital, 2.4 .

Distribución. Hasta ahora, recolectada solamente en San Felipe Orizatlán, Hidalgo, México.

Habitat. Microcentrum tecactli n. sp., es arbórea, recolectada en bosque de encino.

Etimología. El nombre de esta especie "tecactli" es una palabra Náhuatl que significa "huasteco (a)", región del Estado de Hidalgo México, donde se recolectaron los ejemplares.

Señales acústicas. Se grabaron dos tipos de señales acústicas, el canto de llamado "siseo" y secuencias de pulsaciones (tics) producidas en grupos de trenes de pulsaciones-pares (Figs. 33, 35). Ambas señales se producen durante el día y la noche, aunque por la noche hay mayor actividad acústica. La señal de llamado está compuesto por un tren de pulsaciones $=1$ sílaba (Fig. 33), se produce por largos periodos (hasta $1 \mathrm{~h}$ continua) y tiene una duración promedio de $155 \pm 38 \mathrm{~ms}$ ( $78-212 \mathrm{~ms}$ ) (3 machos; $\mathrm{n}=29$ muestras de un min cada una). El intervalo entre sílabas es de $1483 \pm 364 \mathrm{~ms}(115-2394)$. La primera parte de la sílaba, en promedio primeros $23 \mathrm{~ms}$ (20-24; $\mathrm{n}=15$ sílabas) es de mayor intensidad, enseguida la intensidad disminuye gradualmente. El rango de frecuencia (Fig. 34) es de 4 a $>30 \mathrm{kHz}$, el espectro de frecuencia dominante es de 4 a $22 \mathrm{kHz}$ y la frecuencia pico ( $f$ i) es de $4-16 \mathrm{kHz}$; la energía que se libera decae notoriamente después de $30 \mathrm{kHz}$. La taza de repetición de sílabas es de 48 44.36/min (43-55; n=10 muestras de un min cada una).

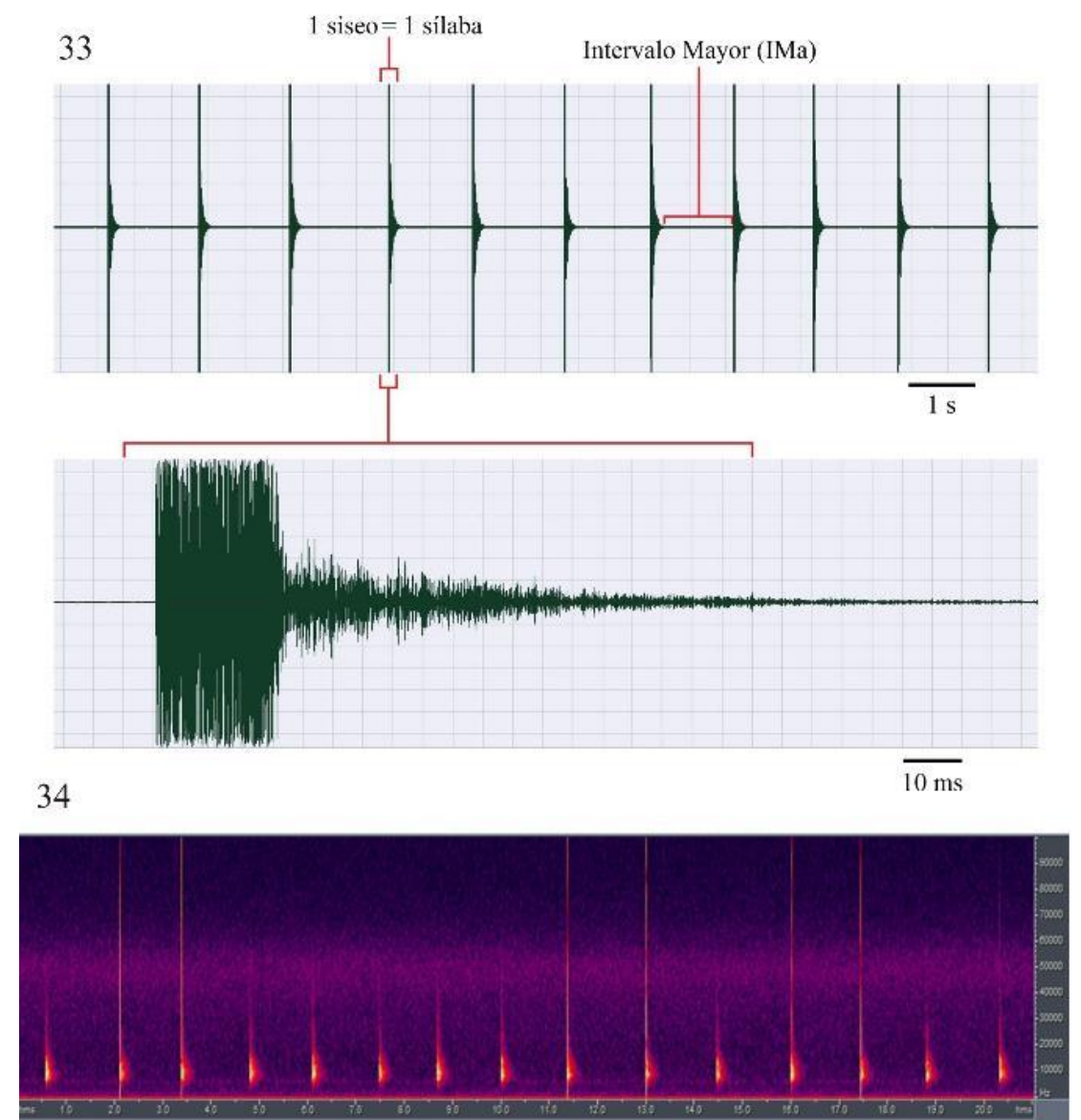

Figura 33-34. Microcentrum tecactli n. sp. San Felipe Orizatlán, Hidalgo, México. 33) Oscilograma de la señal acústica de llamado "siseo". 34) Espectro de frecuencia y fi pico en la señal acústica de siseo. 
En el segundo tipo de señal acústica que se registró (Fig. 35), secuencias-pares de pulsaciones/tics (= dos frases), la duración promedio de éstas es de $7.53 \pm 0.16 \mathrm{~s} \mathrm{(} \mathrm{n}=5$ secuencias-pares de pulsaciones/tics), este tipo de señal se produce ocasionalmente, en referencia al canto de llamado que se produce por horas una vez que los machos están sexualmente maduros. La duración promedio de las frases 1 y 2 es igual en cada secuencia, $2.3 \pm 0 \mathrm{~s}$. El número de pulsaciones por frase es también igual en ambas frases: $29 \pm 0$ en cada secuencia. El intervalo mayor entre secuencias-pares de pulsaciones/tics es de $5.37 \pm 2.75 \mathrm{~s}(\mathrm{n}=5$ secuencias-pares de pulsaciones/tics). El intervalo menor, entre las frases 1 y 2 , es de $1.42 \pm 0.12 \mathrm{~s} \mathrm{(1.27-}$ 1.51). El rango de frecuencia (Fig. 36) es de 4 a $>30 \mathrm{kHz}$, después de $30 \mathrm{kHz}$ la cantidad de energía es mínima. El espectro dominante de frecuencia es de 4 a $22 \mathrm{kHz}$ y la frecuencia pico (fi) es de $4-16 \mathrm{kHz}$. La tasa de repetición de secuencias-pares de pulsaciones es de $0.1 / \mathrm{s}(6 / \mathrm{min})$, en cuatro secuencias-pares de pulsaciones la tasa de repetición de pulsaciones (tics)/s en las frases 1 y 2 fue igual, 12.6.

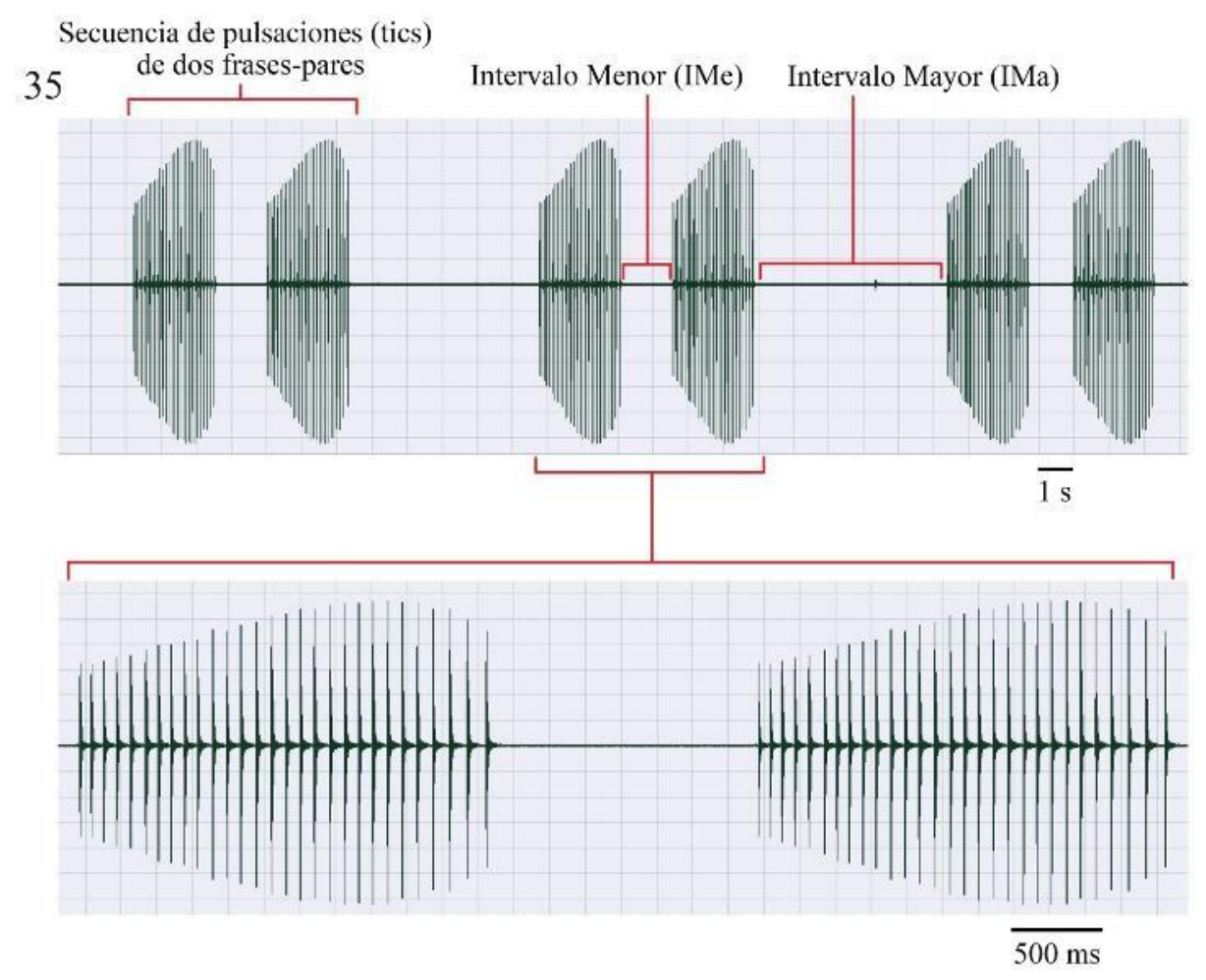

\section{6}

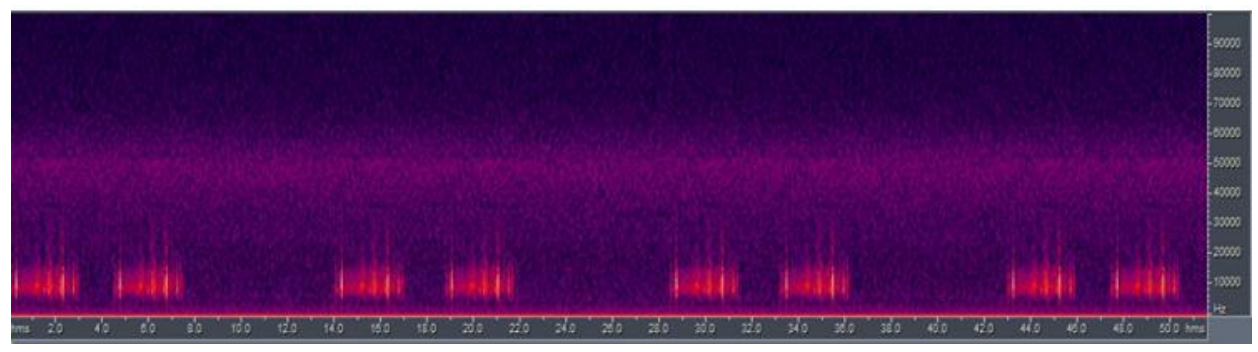

Figura 35-36. Microcentrum tecactli n. sp. San Felipe Orizatlán, Hidalgo, México. 35) Oscilograma de secuencias-pares de pulsaciones (tics). 36) Espectro de frecuencia y fi pico en secuencias-pares de pulsaciones (tics). 

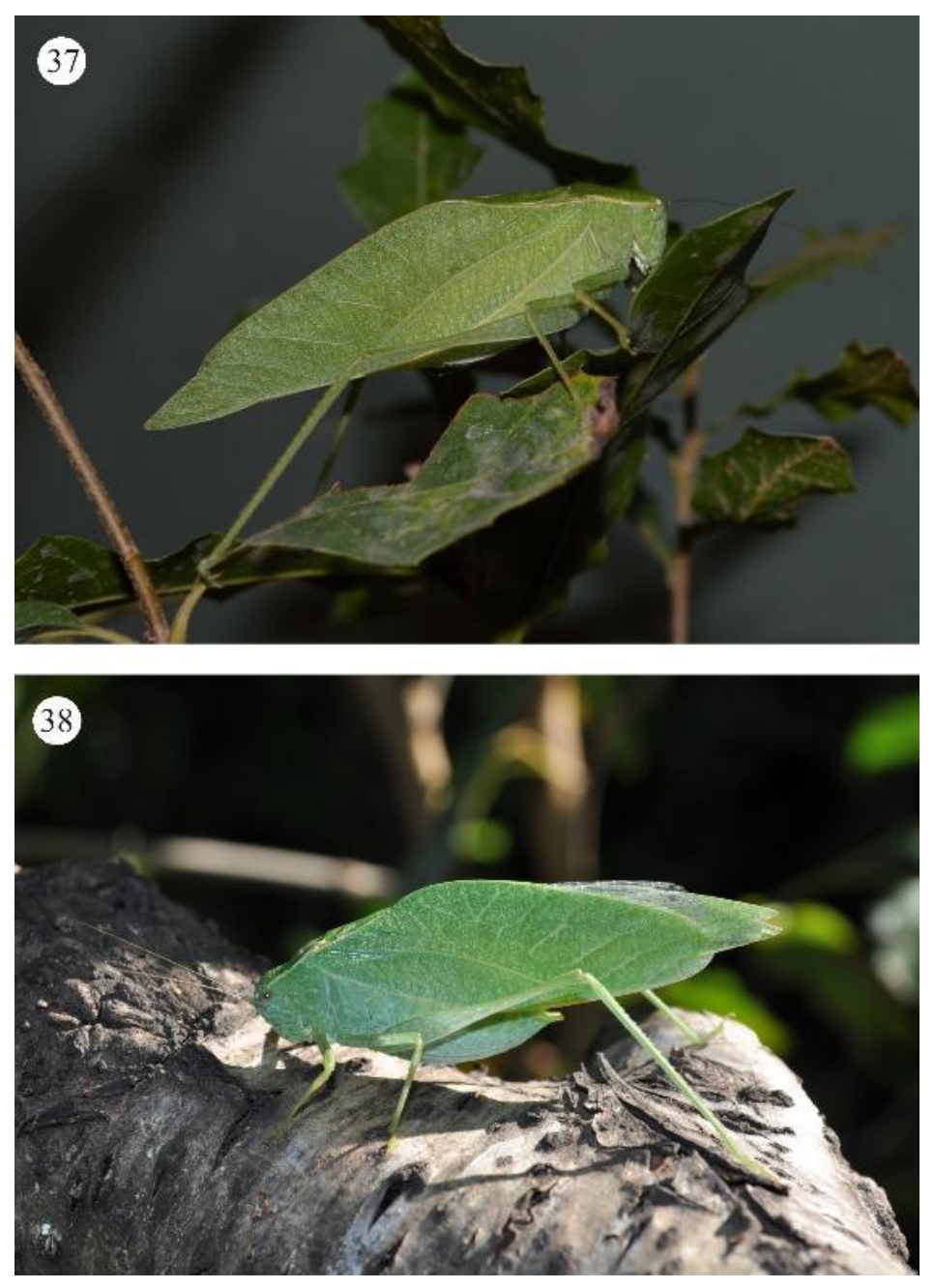

Figuras 37-38. 37) Microcentrum tecactli n. sp. Macho, en hábitat natural. 07.X.2015. San Felipe Orizatlán, Hidalgo, México. 38) Microcentrum rhombifolium. Macho, en hábitat natural. 26.VIII.2015. Tula, Tamaulipas, México.

\section{DISCUSIÓN}

Microcentrum tecactli n. sp., y $M$. rhombifolium ocurren simpátricamente en la Huasteca hidalguense, Barrientos-Lozano et al., (2013) han recolectado M. rhombifolium en repetidas ocasiones en la región noreste del país, incluyendo la huasteca en el Estado de Hidalgo. Microcentrum tecactli n. sp., puede confundirse con $M$. rhombifolium. No obstante, ambas especies pueden separarse en base a los caracteres de diagnóstico siguientes: en machos, el margen anterior del fastigio del vertex, vista frontal, es fuertemente ondulado (casi recto en $M$. rhombifolium), ángulos laterales anteriores del fastigio del vertex muy abultados (moderadamente abultados en M. rhombifolium); fastigio frontal con margen anterior irregular (semi-circular en $M$. rhombifolium), porción media proyectándose hacia la ondulación media del fastigio del vertex; longitud del peine estridulador $4.2 \mathrm{~mm}$ con $c a 62$ dientes $v s, 4 \mathrm{~mm}$ con $c a 55$ dientes en $M$. rhombifolium; plato subgenital-vista ventral- más largo, $2.4 \mathrm{~mm} v s 1.8 \mathrm{~mm}$ en $M$. rhombifolium, emarginación distal en forma de "u" más amplia que en $M$. rhombifolium, estilos más cortos y divergentes (casi rectos en $M$. rhombifolium); los cercos de $M$. tecactli son basalmente un poco más gruesos y el ápice más angosto; la genitalia interna es diferente (Figs. 39a, b vs 40a, b). 

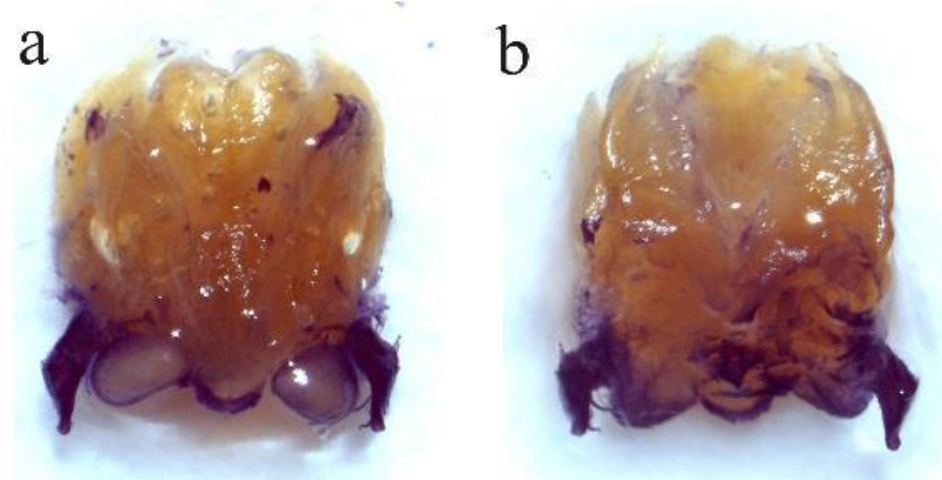

Figura 39. Microcentrum tecactli $\mathrm{n}$. sp. Macho, genitalia interna: a) vista dorsal, b) vista ventral
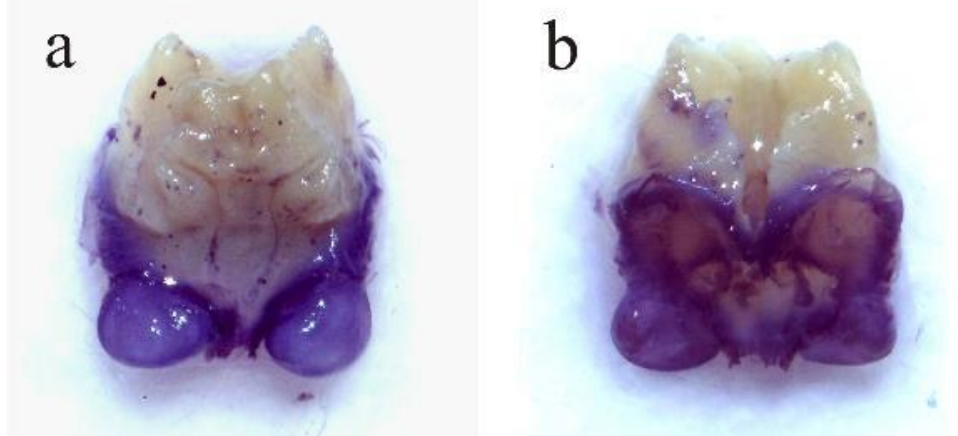

Figura 40. Microcentrum rhombifolium. Macho, genitalia interna: a) vista dorsal, b) vista ventral.

Hembras, el ovipositor es más largo $(5.8 \mathrm{~mm} v s 5.2 \mathrm{~mm})$ y más ancho $(2.3 \mathrm{~mm} v s 1.9 \mathrm{~mm})$, el plato subgenital distalmente emarginado en " $\mathrm{v}$ " vs., redondo en M. rhombifolium. Al analizar la señal acústica de M. tecactli n. sp., nos percatamos que los parámetros temporales, el rango de frecuencia, la fi pico y la tasa de repetición de sílabas y secuencias de pulsaciones por segundo y/o por min no coinciden con los reportados para M. rhombifolium (Spooner, 1968; Nickle, 1976; Walker, 2005). En el canto de llamado de M. tecactli n. sp., las sílabas "siseos" tienen una duración promedio de $155 \pm 38 \mathrm{~ms}$ (78-212 ms), con una taza de repetición de 48 $44.36 / \mathrm{min}(43-55 ; \mathrm{n}=10 \mathrm{~min})$; mientras que las sílabas/siseos en el canto de llamado de $M$. rhombifolium duran en promedio $25 \mathrm{~ms}(22-30)$, con una tasa de repetición de $1=3 \mathrm{~s}$ ( 2 a 4.1), es decir 20/min (40-82) (Spooner, 1968). El rango de frecuencia para M. tecactli $\mathrm{n}$. sp., es de 4 a $>30$ $\mathrm{kHz}$, el espectro de frecuencia dominante es de 4 a $22 \mathrm{kHz}$ y la frecuencia pico $(f i)$ es de 6-16 kHz. De acuerdo con Nickle (1976) en M. rhombifolium el espectro de frecuencia dominante es de 6 a $12 \mathrm{kHz}$ y la fi pico 9.2 a $9.5 \mathrm{kHz}$. El segundo tipo de señal acústica que se registró para $M$. tecactli n. sp., secuenciaspares de pulsaciones/tics, es diferente de las secuencias de pulsaciones (tics) de M. rhombifolium, ya que en la última especie las secuencias de pulsaciones son individuales (un grupo de tren de pulsaciones) no en pares (Spooner 1968, Nickle 1976). Adicionalmente, el rango de frecuencia (4 a >30 kHz), el espectro dominante de frecuencia (4 a $22 \mathrm{kHz}$ ), y la frecuencia pico (fi) $(6-16 \mathrm{kHz})$ de $M$. tecactli n. sp., son diferentes de los de $M$. rhombifolium. De igual manera, ambas especies difieren en el número de pulsaciones (tics) por frase, para M. tecactli n. sp., se registraron 29 en cada frase a una tasa de repetición de 12.6 pulsaciones/s por frase (igual número en las frases 1 y 2). Para $M$. rhombifolium se reporta un promedio de 22 a 25 pulsaciones por serie/frase a una tasa de repetición de 9 pulsaciones (tics)/s (Nickle 1976). De acuerdo con Nickle (1976) cada pulsación corresponde al golpe de un diente del peine estridulador vs., el plectro. Un macho puede estar emitiendo la señal de llamado y de pronto emitir la(s) secuencia(s) de pulsaciones (tics). Inicialmente se pensó que este podría ser el canto de cortejo. Sin embrago, el macho produce esta señal espontáneamente cuando está solo, no en contacto con hembras u 
otros machos, como lo indican Spooner $(1964,1968)$ y Nickle (1976). En M. tecactli n. sp., ambos tipos de señal tienen el mismo rango de frecuencia $(4 \mathrm{a}>30 \mathrm{kHz})$ y similar fi pico $(6-16 \mathrm{kHz})$. Ambos tipos de señal intervienen en la búsqueda de pareja y el apareamiento. No obstante, no es totalmente clara la función de la primera señal "siseo", Spooner (1968) y Nickle (1976) reportan que las hembras solo responden acústicamente, con uno a dos tics, a la secuencia de pulsaciones de los machos, lo cual ocurre $249 \pm 27$ s después del último tic de la secuencia del macho. Spooner (1968) hace referencia a un posible par de funciones: a) el siseo tiene una función de territorialidad, b) mantiene a hembras receptivas cerca del macho que emite el siseo. Otro aspecto relevante en el sistema acústico de comunicación de Microcentrum spp., es la intensidad de la señal. En el caso de M. tecactli n. sp., los primeros $23 \mathrm{~s}$ de la señal de llamado son de mayor intensidad. Spooner (1968) muestra experimentalmente que las hembras de M. rhombifolium responden acústica y físicamente (caminando hacia la fuente emisora del sonido) solo cuando el siseo de los machos es de $55 \mathrm{~dB}$, no obteniendo respuesta a 75 y $90 \mathrm{~dB}$. Se documenta la variación inter-específica en parámetros temporales y frecuencia entre $M$. tecactli $\mathrm{n}$. sp., y $M$. rhombifolium, lo cual ocurre frecuentemente en Phaneropterinae (Nickle \& Carlysle, 1975; Spooner, 1995; Heller et al., 2015, 2017).

\section{CONCLUSIONES}

Se describe $M$. tecactli n. sp., endémica hasta ahora, para la región huasteca del Estado de Hidalgo, México; se proporcionan caracteres de diagnóstico para separarla de M. rhombifolium. Se presenta un análisis de las señales acústicas, canto de llamado "siseo" y "secuencias-pares de pulsaciones" que presentan diferencias que claramente distinguen las especies. Ambas señales tienen espectro de frecuencia $(4-30 \mathrm{kHz})$ y $f i$ pico $(6-16 \mathrm{KHz})$ similar. La primera parte de la señal de llamado "siseo", es de mayor intensidad. No es clara la significancia del canto de llamado "siseo", por lo que se sugiere estudiar con mayor detalle el sistema de comunicación acústica de $M$. tecactli n. sp.

AgRAdeCimientos. Se agradece al CONACYT por el apoyo proporcionado a través del proyecto CB/2013/0219979. Al Tecnológico Nacional de México-Instituto Tecnológico de Cd. Victoria, por el apoyo recibido para realizar el trabajo de campo.

\section{LITERATURA CITADA}

Barrientos-Lozano, L., Rocha-Sánchez, A. Y. Buzzetti, F. M., Méndez-Gómez, B. R., Horta-Vega, J. V. (2013) Saltamontes y Esperanzas del Noreste de México. Guía Ilustrada. Dirección General de Educación Superior Tecnológica-Instituto Tecnológico de Cd. Victoria, Tamaulipas, México. 304 pp.

Braun, H. (2015) On the family-group ranks of katydids (Orthoptera, Tettigoniidae). Zootaxa, 3956 (1), $149-150$.

Cigliano, M. M., Braun, H., Eades, D. C., Otte D. (2018) Orthoptera Species File Online. Version 5.0/5.0.http://Orthoptera.SpeciesFile.org.

Fontana, P., Buzzetti, F. M., Mariño-Pérez, R. (2008) Chapulines, Langostas, Grillos y Esperanzas de México. Guía Ilustrada. WBA Handbooks, 1, Verona: 1-272pp.

Fulton, B. B. (1933) Stridulating organs of female Tettigoniidae (Orthoptera). Entomological News, 44, 270-275.

Heller, K. G., Orci, K. M., G. Grein., Ingrisch, S. (2004) The Isophya species of Central and Western Europe (Orthoptera: Tettigonioidea: Phaneropteridae). Tijdschrift Voor Entomologie, 147, 237-258.

Heller, K. G., Hemp, C., Ingrisch, S., Liu, C. (2015) Acoustic Communication in Phaneropterinae (Tettigonioidea), A Global Review with Some New Data. Journal of Orthoptera Research, 24 (1), 7-18. DOI: http://dx.doi.org/10.1665/034.024.0103

Heller, K. G., Ingrisch, S., Liu, C. X., Shi, F. M., Hemp, C., Warchaowska-Liwa, E., Rentz, D. C. F. (2017) Complex songs and cryptic ethospecies: the case of the Ducetia japonica group (Orthoptera: 
Fernández-Azuara et al.: Microcentrum tecactli n. sp. Phaneropteridae

Tettigonioidea: Phaneropteridae: Phaneropterinae). Zoological Journal of the Linnean Society, XX, $1-22$.

Nickle, D. A., Carlysle, T. C. (1975) Morphology and function of female sound-producing structures in Ensiferan Orthoptera with special emphasis on the Phaneropterinae. International Journal of Insect Morphology \& Embryology, 4 (2), 159-168.

Nickle D. A. (1976) Interspecific differences in frequency and other physical parameters of pair-forming sounds of bush katydids (Orthoptera: Tettigoniidae: Phaneropterinae). Annals of the entomological Society of America, 69, 1136-1144.

Morris G. K., Walker, T. J. (1976) Calling songs of Orchelimum meadow katydids (Tettigoniidae): I. Mechanism, terminology, and geographic distribution. Canadian Entomologist, 108, 785-800.

Mugleston, J. D., Song, H., Whiting, M. F. (2013) A century of paraphyly: A molecular phylogeny of katydids (Orthoptera: Tettigoniidae) supports multiple origins of leaf-like wings. Molecular Phylogenetics and Evolution, 69, 1120-1134.

Ragge, D. R., Reynolds, W. J. (1998) The Songs of the Grasshoppers and Crickets of Western Europe. Colchester, Harley Books, 591 pp.

Spooner, J. D. (1964) The Texas bush katydid-its sounds and their significance. Animal Behaviour, 12, $235-244$.

Spooner, J. D. (1968) Pair-forming acoustic systems of Phaneropterinae katydids (Orthoptera, Tettigoniidae). Animal Behaviour, 16, 197-212.

Spooner, J. D. (1995) Pair-forming Phonotaxic strategies of Phaneropterinae katydids (Tettigoniidae: Phaneropterinae). Journal of Orthoptera Research, 4, 127-129.

Stumpner, A., Dann, A., Schink M., Gubert, S., Hugel, S. (2013) True katydids from Guadeloupe: Acoustic signals and functional considerations of song production. Journal of Insect Science, 13.157, 1-16. Available online: http://www.insectscience.org/13.157

Walker, T. J. (2005). Singing insects of North America (SINA). Available at: https://entnemdept.ifas.ufl.edu/walker/Buzz/index.htm (accessed on March 2018). 